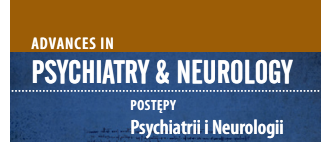

Correspondence to:

I Putu E. Widyadharma

Department of Neurology

Faculty of Medicine

Udayana University

Sanglah General Hospital

Bali, Indonesia

e-mail: eka.widyadharma@unud.ac.id

Submitted: 24.12 .2019

Accepted: 03.06.2020

\section{THE ROLE OF OXIDATIVE STRESS, INFLAMMATION AND GLIAL CELL IN PATHOPHYSIOLOGY OF MYOFASCIAL PAIN}

\author{
I Putu E. Widyadharma \\ Department of Neurology, Faculty of Medicine, Udayana University, Sanglah \\ General Hospital, Bali, Indonesia
}

\begin{abstract}
Purpose: The aim of this article is to explain the role of oxidative stress, inflammatory responses, and glial cell in the pathophysiology of myofascial pain. Therefore the management of myofascial pain can be optimally done by clinicians through blockage of each biomarker in a specific pathway.

Views: Myofascial pain is often one of the reasons for patients to visit the doctor with a prevalence of approximately 21-30\%. Overused muscle can lead to myofascial trigger points. Activities that cause ongoing muscle contraction can cause an increase in metabolic stress and decreased blood flow resulting in the imbalance of oxidative-antioxidant. Malondialdehyde is one of the biomarkers of oxidative stress. This process also can increase the release of neuropeptides, cytokines, and inflammatory substances. Prostaglandins, especially prostaglandin E2 (PGE2), can increase vascular permeability and cell proliferation that binds to sensory neuron receptors, which facilitate sensitization to the pain nerve. Astrocytes are the most abundant cell type in the central nervous system, which plays an essential role in the induction and persistence of pain. In ischemic conditions, astrocytes will alternate and turn into reactive astrogliosis. This condition will increase the level of glial fibrillary acidic protein.

Conclusions: The exact pathophysiology of myofascial pain is not thoroughly clear. Hence, some studies found the total levels of oxidative stress were higher in patients with myofascial pain. Malondialdehyde, PGE2, and GFAP as the biomarkers from those factors are increased in patients with myofascial pain.
\end{abstract}

Key words: myofascial pain, malondialdehyde, prostaglandin E2, glial fibrillary acidic protein.

\section{INTRODUCTION}

Myofascial pain is one of the health problems that attract the attention of clinicians and has also caused confusion and errors in recent decades. Myofascial pain has been experienced by almost everyone at around $85 \%$, with an estimated prevalence of 21-30\% [1-3]. Myofascial pain consists of autonomic, motoric, and sensory symptoms that involve both the muscles and surrounding connective tissue (fascia). Myofascial pain is caused by myofascial trigger points, which are located on the taut muscle band [4-14]. The shortening of the contracted taut muscle band results in movement restriction and pain. Myofascial pain can be classified into acute myofascial pain if there are 1 or 2 regions of local pain that can disappear after a few weeks and chronic myofascial pain if the pain persists for more than six months. The pain characteristic of the chronic myofascial pain is generalized pain with a poorer prognosis. Acute myofascial pain usually occurs after excessive activity, whereas chronic myofascial pain occurs in patients with stress factors $[6,8]$. There is no certain consensus in diagnosing myofascial pain. However, the most commonly used diagnostic criteria is by Travell and Simons, which consists of five major criteria and three minor criteria. The major criteria are regional pain symptoms, pain characteristics accompanied by referred pain, palpable taut muscle band, focal pain in one point or nodule in the taut muscle band, and restriction of movement or slight muscle weakness. The minor criteria are pain at the palpation of the myofascial trigger point, a local twitching response (LTR) on sudden palpation at the myofascial trigger point, and decreased pain with muscular therapy $[15,16]$.

The clinical presentation of myofascial pain is very complex. The onset and persistence of myofascial pain due to the role of myofascial trigger points can be discovered through physical examination $[2,17,18]$. Myofascial trigger points are abnormal characteristics of nodules 
in the focal areas of hypersensitive muscles, hard in palpation, stiff and pain during physical examination [2-6, 9, 10, 14, 17, 19-21]. According to Travell and Simons, myofascial trigger points can be classified into two types, active and latent myofascial trigger points. In an active myofascial trigger point, the pain occurs spontaneously without being manipulated or exacerbated by palpation in the surrounding tissue and or spreads according to the location of referred pain, with motor or autonomic symptoms. The referred pain is caused by central sensitization and makes the disease more complex $[6,8,14$, $16,22]$. Whereas the latent myofascial trigger points, have symptoms similar to the active type through pain stimulation when the area of skeletal taut muscle band is being compressed/palpated, or with a local muscle twitching response and referred pain. The active myofascial trigger point has a lower pain threshold than at the latent myofascial trigger point $[2-4,6,9,14-16,18,19,21-23]$. Continuous peripheral nociceptive input activates dorsal horns through the release of substance P. This process causes neuroplastic changes (increased excitability) in the central nervous system and causes central sensitization, which results in allodynia and hyperalgesia from stimulation of activity in the region of the brain to process sensory discrimination $[8,9,15,21]$.

According to Gerwin, there are several diagnostic criteria of myofascial pain, which are the finding of the tender points in a taut muscle band, the presence of LTR and or pain during palpation, restriction of movement, the emerging of pain symptoms at the active myofascial trigger point, regional muscle weakness, and autonomic symptoms $[5,15]$. Simon revealed the hypothesis of the occurrence of the myofascial trigger point is that the excessive released of acetylcholine, which stimulates an increase in the strain on muscle fibers at the myofascial trigger point [2]. Also, according to Travell and Simon, vasoconstriction will cause the local hypoxia/ ischemia. This vasoconstriction occurs due to increased calcium in tissues when the sarcoplasmic reticulum rupture occurs $[2,20,24]$. Decreased oxygen will disrupt mitochondrial metabolism. In this situation, the reduction of ATP will cause an energy crisis and distress in the tissue $[2,6,8,10,14,25,26]$. Therefore anaerobic metabolism will occur that produces lactic acid, reducing the intramuscular $\mathrm{pH}$ [14].

The proliferation of microtubules is associated with myofascial trigger points and myofascial pain. Examination using Doppler ultrasound reveals a hypoechoic focal area with a heterogeneous echotexture. In elastography, vibration amplitude decreases, and there is an entropy area with rigid tissue compared to the surrounding tissue. This shows an increase in density, which indicates the contraction or proliferation of proteins $[2,5,9]$. Two mechanisms that cause pain at the myofascial trigger point, such as the involvement of ASIC3 nociceptors (acid-sensing ion channels) and TRPV1 (transient receptor potential) channels, which are known as capsaicin receptors on pain nerves [2]. ASIC is associated with myalgia without any damage to muscles [9]. The decrease in $\mathrm{pH}$ (below 5.0) that occurs due to local ischemia at the myofascial trigger point is more than sufficient to cause excitation of muscle nociceptors, including the ASIC3 and TRPV1 canal nociceptors $[2,14]$.

\section{ROLE OF OXIDATIVE STRESS IN PATHOPHYSIOLOGY OF MYOFASCIAL PAIN}

Oxidative stress is a disproportion of reactive oxygen species (ROS) and the capability of biological systems to detoxify dangerous reactive intermediates [27, 28]. Conditions of oxidative stress will increase levels of free calcium and iron ions in cells that cause cell damage and even cell death. If free radical production increases in certain levels in the body, the enzyme defensive system will fail, and there will be oxidative stress. Superoxide radicals are a form of ROS formed in the brain. Superoxide dismutase (SOD) helps the body to get rid of superoxide radicals by transforming them into hydrogen peroxide $\left(\mathrm{H}_{2} \mathrm{O}_{2}\right)$, and then they are catalyzed into water and oxygen. In addition, glutathione peroxidase also plays a role in the removal of $\mathrm{H}_{2} \mathrm{O}_{2}$ from the body [27-29]. Activities that cause ongoing muscle contraction can cause an increase in metabolic stress and decreased blood flow resulting in persistence of the myofascial trigger point. Vasoconstriction, due to the increased calcium levels, causes ischemia and produces free oxygen radicals that damage endothelial and cause further ischemia, which plays a role in the pathophysiology of myofascial pain [2, 24]. Cascade of X-ROS signaling (stretch-activated ROS production) will change the shape of microtubules to activate NADPH (nicotinamide adenine dinucleotide phosphate) oxidase (NOX2) to produce ROS which will be oxidized to RyRs (ryanodine receptors) and increase the sarcoplasmic reticulum to release $\mathrm{Ca}^{2+}$ ions. Within the skeletal muscle, $\mathrm{X}$-ROS will sensitize the $\mathrm{Ca}^{2+}$ permeable sarcolemmal transient receptor potential (TRP) channel. This process is critical in maintaining the sarcoplasmic reticulum when muscle contractions occur continuously [2]. This mechanism shows that excessive contraction is associated with stress that involves cytoskeletal microtubules to activate NADPH oxidase to produce ROS. Calcium release can stimulate contractions to form a myofascial trigger point. In this condition, there will be a decrease in glutathione levels and an increase in glutathione disulfide levels, which is oxidized glutathione. The main function of glutathione as an anti-oxidant is to detoxify free radicals and reactive oxygen (ROS) in cells [2, 29]. In several studies, high serum malondialdehyde levels were 
found with low superoxide dismutase and impaired oxidative-antioxidant balance [24].

Malondialdehyde consists of 3 carbons which can be produced either through the oxidation of polyunsaturated fatty acids or through arachidonic acid metabolism. Malondialdehyde production through an enzymatic process occurs when biosynthesis of thromboxane A2 (TXA2), which is an active metabolite, is formed through arachidonic acid metabolism with thromboxane A2 synthase and also results from the breakdown of prostaglandin endoperoxide $\left(\mathrm{PGH}_{2}\right)$ to 23-hydroxyheptadecatrienoate (HHT), while the production of malondialdehyde is from a non-enzymatic process that occurs through lipid peroxidation. Lipid peroxide is derived from polyunsaturated fatty acids $\left(\mathrm{C}_{7} \mathrm{H}_{16} \mathrm{O}_{4}\right)$ which has unstable properties and can be converted to malondialdehyde (MDA). Lipid peroxidation occurs due to the formation of substances resulting from cellular damage $[27,28,30]$. Arachidonic acid induces platelets to form a considerable amount of malondialdehyde. Malondialdehyde is a biomarker when oxidative stress occurs and can be used to diagnose diseases. Malondialdehyde is the most reactive form of dialdehyde [27, 28]. Malondialdehyde can be metabolized enzymatically or can react in cells and tissues. Malondialdehyde oxidation is carried out by mitochondrial aldehyde dehydrogenase. Then the malondialdehyde will be decarboxylated to form acetaldehyde, which is oxidized by aldehyde dehydrogenase to produce acetate, $\mathrm{H}_{2} 0$, and $\mathrm{CO}_{2}$. As far as the author's knowledge, there is not any studies yet that discuss the exact time of the oxidation of acetaldehyde by the enzyme aldehyde dehydrogenase in detail [30].

Besides, excessive reactive oxygen production at the myofascial trigger point mechanism will activate TRP (transient receptor potential) channels, namely TRPM2, TRMP7, TRPC5, TRPV1, and TRPA1, but only TRPV1-4, TRPA1, and TRMP8 are associated with pain. The active type myofascial trigger point is located closer to the pain channel receptors, which have high levels of protons and ROS, which can activate the ASIC 3 and TRPV1 channels. Whereas at the latent type of myofascial trigger point, the location is further from the pain channel receptors so that the amount of ROS, which is a large unstable molecule, can decrease and not enough to be able to activate the pain nociceptors spontaneously. Therefore, in experimental studies, it has been proven that suppression of cells can activate stretch channels which then increase the myoplasmic calcium through the X-ROS mechanism so that when there is a palpation/compression in the myofascial trigger point area, there will be an increase in ROS levels sufficient to activate canal TRPV1 and cause pain [2]. In a study done by Irfan Koca et al., it was found that there were statistically significant differences between patients and controls, namely the low levels of total antioxidant capacity (TAC) in patients with myofascial pain
( $p=0.007$ ), with total levels of oxidative stress (total oxidative stress - TOS) was higher in patients with myofascial pain $(p=0.045)$. From these studies, it can be concluded that myofascial pain is associated with a disturbance of oxidative-antioxidative balance [24].

\section{ROLE OF INFLAMMATION IN PATHOPHYSIOLOGY OF MYOFASCIAL PAIN}

Prolonged muscle contraction, ischemia/hypoxia, metabolic disorders, and cell stress also lead to increased release of neurotransmitters, inflammatory cytokines, and myokines, which necessary in the pathophysiology of myofascial pain [2, 25, 31]. Damage to these muscles cause the release of neuropeptides, cytokines, and inflammatory substances such as potassium, bradykinin, cytokines, tumor necrosis factor, interleukin $1 \beta$, norepinephrine, protons, prostaglandins, ATP and substances $\mathrm{P}$ that can stimulate nociceptors in the muscle thereby releasing CGRP (calcitonin gene-related peptide) $[2,8,9$, $15,18,25]$. Mast cells will be degranulated by substance $P$ and release serotonin, histamine, and upregulation of anti-inflammatory cytokines (IL-4 and IL-10) and also the pro-inflammatory factors (TNF- $\alpha$ and IL-6). The release of CGRP and substance $\mathrm{P}$ as a vasodilator cause more capillary compression. TNF- $\alpha$ is the only cytokine that is stored in mast cells and will be released immediately when mast cell degranulation occurs. The release of TNF- $\alpha$ will trigger norepinephrine production. Studies found an increase in both serotonin and norepinephrine in patients with active myofascial trigger points $[9,18]$. The histopathological examination obtained from the multiple muscle fibers at the myofascial trigger point revealed shortened sarcomeres regionally as well as excessive release of acetylcholine [18]. Hypotheses expressed by Simons and further explained by Gerwin et al. stated that CGRP would inhibit acetylcholinesterase. Increase of the acetylcholine receptors and increase of acetylcholine release (acetylcholine leakage) will lead to prolonged contraction of local muscle fibers, known as myofascial trigger points. Continuous input at myofascial trigger point will cause apoptosis of inhibitory neurons at the segmental level and will sensitize neurons at dorsal horn causing hyperalgesia, allodynia, and prolonged pain $[2,5,14,18]$. However, to maintain contractions, the shortening of sarcomeres requires high oxygen levels. This will increase the metabolic demand that leads to ischemia and cause more severe local hypoxia [18]. Acidic $\mathrm{pH}$ will stimulate the further release of bradykinin when local ischemia and inflammation occur. Therefore, it can be proven that acidic $\mathrm{pH}$ is associated with the onset of myofascial pain. Gerwin et al. said that a low $\mathrm{pH}$ could inhibit acetylcholinesterase activity, thereby caus- 
ing more acetylcholine to be released in the synaptic cleft and lead to prolonged sarcomere contractions. This process indicates the occurrence of the phenomenon of taut muscle band (focal muscle contraction modulated by afferent muscle spindles) $[9,14,18,26]$.

Although there are low levels of serotonin in the tissues, serotonin receptor depolarization does not occur. Low serotonin levels will sensitize neurons to activate other factors, such as prostaglandins. Prostaglandins, especially prostaglandin E2, will sensitize pain neurons. ATP will activate P2X3 receptors [2]. If cell damage occurs, prostaglandins that originate from arachidonic acid conversion, which is become an unstable intermediate (prostaglandin $\mathrm{H}_{2}\left[\mathrm{PGH}_{2}\right]$ ), will be excreted via the cyclooxygenase (COX-1 and COX-2) catalysis pathway. PGE synthase will catalyze $\mathrm{PGH}_{2}$ into mPGES-1, mPGES-2, and cPGES. Then the PGI synthase, prostaglandin F synthase, and thromboxane synthase are catalyzed into prostaglandin E2 (PGE2), prostacyclin $\left(\mathrm{PGI}_{2}\right)$, prostaglandin $\mathrm{F}_{2 \alpha}$ and thromboxane $\mathrm{A}_{2}$ (TXA ${ }_{2}$ ), respectively [32-35]. PGE2 plays a role in various processes, such as controlling vascular tonus, increasing vascular permeability, and cell proliferation. PGE2 is the most produced mediator from prostaglandin synthesis, especially in areas that are inflamed $[32,36,37]$. PGE2 plays an important role in inflammatory symptoms such as fever, edema, and pain sensations in both acute and chronic pain $[32,34,36]$.

PGE2 can reduce the nociceptive threshold, causing pain. The subtypes of PGE2 receptors consist of $\mathrm{EP}_{1}, \mathrm{EP}_{2}$, $\mathrm{EP}_{3}$, and $\mathrm{EP}_{4}$. All PGE2 receptor subtypes are $\mathrm{G}$ protein-coupled receptors (GPCRs) $[33,35,37]$. Subtype EP works through the PLC/IP3 and DAG/PKC pathways by binding to $\mathrm{Ca}^{2+}$ intracellular, which causes hyperalgesia. Subtypes $\mathrm{EP}_{2}, \mathrm{EP}_{4}$ together with prostacyclin receptors working through the AC/cAMP/PKA pathway, while the $\mathrm{EP}_{3}$ subtype provides an inhibitory effect. Through the $\mathrm{EP}_{3}$ receptor subtype, PGE2 can stimulate mast cell degranulation, thereby causing vascular hyperpermeability and forming edema [35-37]. In the study, it was said that the $\mathrm{EP}_{4}$ subtype was the most dominant in inducing neurons in the dorsal horn to cause hypersensitivity in inflammatory pain. PGE2 can modulate pain transduction and improve the function of TRPV1 to the capsaicin response $[33,34]$. Prostaglandins can also increase the effects of other chemical mediators such as 5-HT, bradykinin and release of neuropeptides such as substance $\mathrm{P}$ and CGRP, thereby increasing the sensitivity of mechanical receptors. Bradykinin can sensitize muscle nociceptors in mechanical stimuli and further enhance the synthesis and release of PGE2. All these processes occur in the peripheral sensitization that causes hyperalgesia [35, 38]. At the peripheral, PGE2 binds to sensory neuron receptors, thereby increasing the response of the ion channel, which facilitates sensitization to the pain nerve. In the central nervous system, PGE2 can be synthesized through peripheral inflammatory responses that induce phosphorylation of glycinergic receptors in the dorsal horn of the spinal cord $[34,39]$.

\section{ROLE OF GLIAL CELLS IN PATHOPHYSIOLOGY OF MYOFASCIAL PAIN}

Prolonged nociceptive input can cause maladaptive changes in the central nervous system. Prolonged afferent nociceptive input from the myofascial trigger point activates and sensitizes the neurons in the dorsal horn, which carried by the spinothalamic tract to higher brain centers. Muscle afferent input activates not only the thalamus but also the limbic system, which plays an important role in pain modulation and emotions $[5,21]$. Patients with chronic myofascial pain can cause atrophy of the region in cerebral substantia grisea, which has a role in perception and modulation of pain $[19,21,22]$. The limbic system is very closely related to the body stress response. In a study conducted by Niddam et al., there was an increase in the activity of the limbic system in patients with myofascial pain at trapezius muscle, which corresponds with greater tension and stress [5]. Neural circuits in the central nervous system consist of various cell types, including neurons and glial cells. Glial cell activation and neuro-glial interactions play a part in the mechanism of chronic pain.

Microglia, astrocytes, and oligodendrocytes are three types of glial cells in the central nervous system. Whereas glial cells in the peripheral nervous system consist of schwann cells in the peripheral nerve, satellite glial cells (SGC) in the dorsal ganglia, and the trigeminal ganglia (TG) $[40,41]$. Astrocytes are the most abundant cell type in the central nervous system, which regulates the physiology and pathology in the central nervous system such as regulation of fluids and ions, blood flow control, vascular generation, protection of neurons from toxic damage and others. Through the astrocyte-microglia relationship, astrocytes can modulate the microglia phenotype and phagocytosis [40-42]. Astrocytes play an important part in the induction and persistence of pain [41, 43]. If there is a damage or the presence of dangerous stimuli such as neuroinflammation and ischemia, astrocytes will change in morphology and function, known as reactive astrogliosis [40]. The pro-inflammatory factors such as cytokines, oxidative stress, and free radicals found in patients with myofascial pain will initiate the reactive astrocytes. This initiation of reactive astrocytes occurs through the nuclear factor kappa-B (NFkB) pathway. Astrocyte activation and release of IL-1 $\beta$ take part in the mechanism of hyperalgesia. These cytokines and chemokines act in the induction and persistence of pain. The activation of astrocytes has been investigat- 


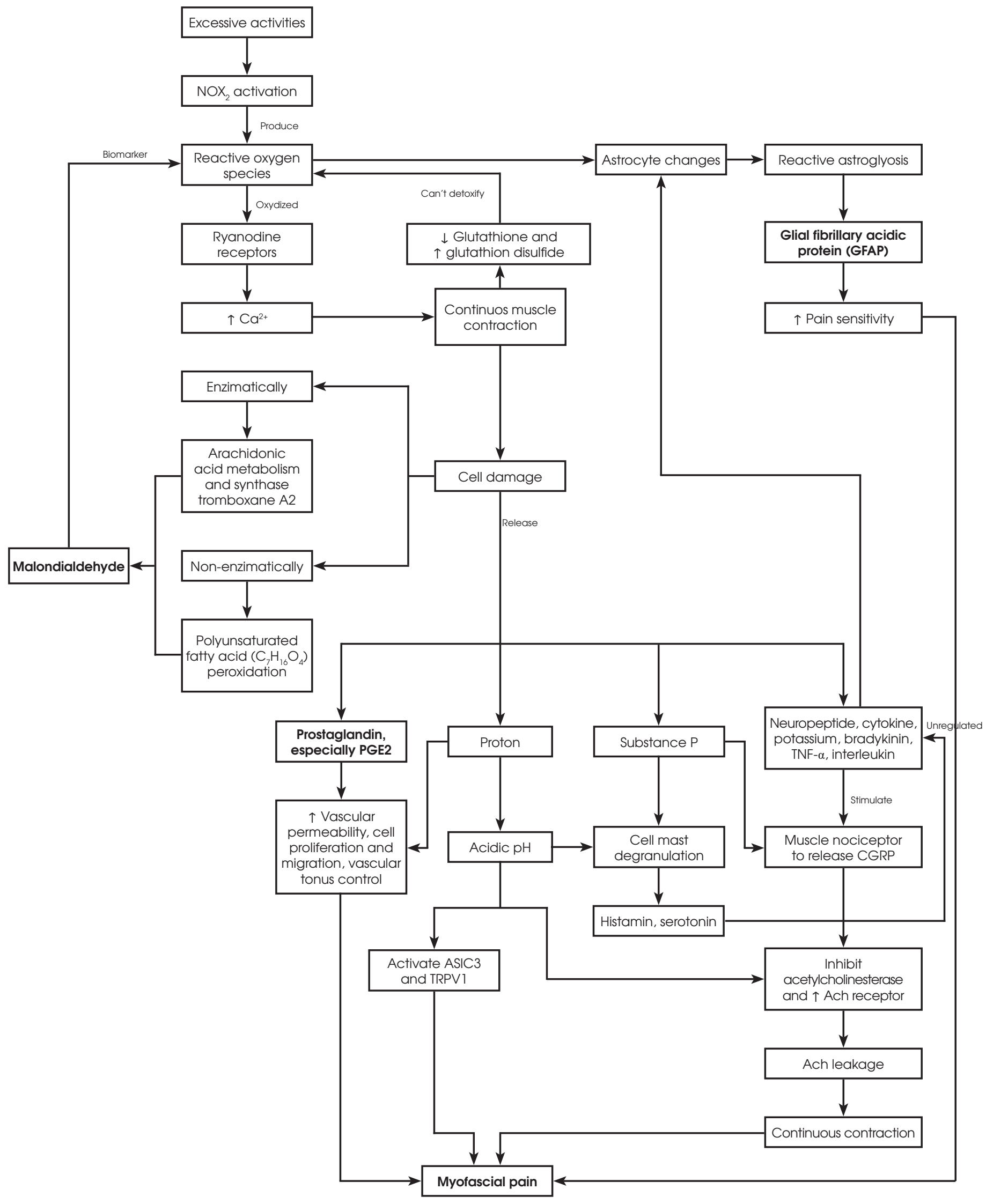

Figure I. Pathophysiology of myofascial pain 
ed in relation to the pathogenesis of pain through the release of pro-inflammatory and pro-algesic mediators such as TNF- $\alpha$, IL-1 $\beta$, and IL-6 [42-44].

Active astrocytes can develop and maintain chronic pain by releasing some substances. These active astrocytes can also regulate emotions in the brain region (primary somatosensory cortex and anterior cingulate cortex, hippocampus, prefrontal, medial cortex) associated with emotional alteration when chronic pain occurs [40]. Pain syndrome is associated with glial cell activation, with an increase in glial cell markers such as IBA1 and glial fibrillary acidic protein (GFAP) with morphological changes. When reactive astrogliosis occurs, the naive astrocytes will become hypertrophic and increase in the GFAP level. Then, the reactive astrocytes will proliferate and become scar-forming astrocytes. Both forms of astrocytes have high protein markers, namely GFAP, nestin, $\beta$-catenin, and N-cadherin. It also occurs in the synthesis and release of glial cell mediators such as growth factors, cytokines, chemokines, and proteases, which can increase pain sensitivity [40-42]. An increase in GFAP is a biomarker of reactive astrocytes [44].

\section{CONCLUSIONS}

The exact pathophysiology of myofascial pain is not thoroughly clear. Studies reported that oxidative stress, inflammation, and glial cell, especially the astrocyte in the central nervous system, cause the persistence of pain sensation that later on lead to the myofascial trigger point. This illustration is described in Figure I. Malondialdehyde, PGE2, and GFAP as the biomarkers from those factors are increased in patients with myofascial pain. With the comprehensive understanding of these processes, we as doctors could provide optimal and efficient therapy by giving drugs that are specific to each pathway.

\section{Conflict of interest}

Absent.

Financial support

Absent.

\section{References}

1. Agung I, Murdana N, Purba H, Fuady A. Low-level laser therapy and dry needling for myofascial pain syndrome of the upper trapezius muscle: an interventional study. J Phys Conf Ser 2018; 1073: 062045. DOI: 10.1088/17426596/1073/6/062045.

2. Jafri MS. Mechanisms of myofascial pain. Int Sch Res Notices 2014; 2014: 523924. DOI: 10.1155/2014/523924.

3. Shah JP, Thaker N, Heimur J, Aredo JV, Sikdar S, Gerber L. Myofascial trigger points then and now: a historical and scientific perspective. PM R 2015; 7: 746-761.

4. Segura-Pérez M, Hernández-Criado MT, Calvo-Lobo C, Vega-Piris L, Fernández-Martín R, Rodríguez-Sanz D. A multimodal approach for myofascial pain syndrome: a prospective study. J Manipulative Physiol Ther 2017; 40: $397-403$.

5. Shah JP, Heimur J. New frontiers in the pathophysiology of myofascial pain. Pain Pract 2012; 22: 26-33.

6. Tantanatip A, Chang KV. Myofascial pain syndrome (MPS). StatPearls [Internet] 2019: 1-4.

7. Xia P, Wang X, Lin Q, Cheng K, Li X. Effectiveness of ultrasound therapy for myofascial pain syndrome: a systematic review and meta-analysis. J. Pain Res 2017; 10: 545-555.

8. Qureshi NA, Alsubaie HA, Ali GIM. Myofascial pain syndrome: a concise update on clinical, diagnostic and integrative and alternative therapeutic perspectives. Int Neuropsychiatr Dis J 2019; 13: 1-14.

9. Gerwin R. Myofascial pain syndrome: here we are, where must we go? J Musculoskelet Pain 2010; 18: 329-347.

10. Kisilewicz A, Janusiak M, Szafraniec R, Smoter M, Ciszek B, Madeleine P, et al. Changes in muscle stiffness of the trapezius muscle after application of ischemic compression into myofascial trigger points in professional basketball players. J Hum Kinet 2018; 64: 35-45.

11. Rivers WE, Garrigues D, Graciosa J, Harden RN. Signs and symptoms of myofascial pain: an international survey of pain management providers and proposed preliminary set of diagnostic criteria. Pain Med 2015; 16 : 1794-1805.

12. Stecco A, Gesi M, Stecco C, Stern R. Fascial components of the myofascial pain syndrome. Curr Pain Headache Rep 2013; 17: 352.

13. Stecco C, Stern R, Porzionato A, Macchi V, Masiero S, Stecco A, et al. Hyaluronan within fascia in the etiology of myofascial pain. Surg Radiol Anat 2011; 33: 891-896.

14. Bron C, Dommerholt JD. Etiology of myofascial trigger points. Curr Pain Headache Rep 2012; 16: 439-444. 
15. Bourgaize S, Newton G, Kumbhare D, Srbely J. A comparison of the clinical manifestation and pathophysiology of myofascial pain syndrome and fibromyalgia: implications for differential diagnosis and management. J Can Chiropr Assoc 2018; 62: 26-41.

16. Fernández-de-las-Peñas C, Dommerholt J. International consensus on diagnostic criteria and clinical considerations of myofascial trigger points: a delphi study. Pain Med 2018; 19: 142-150.

17. Hwang UJ, Kwon OY, Yi CH, Jeon HS, Weon JH, Ha SM. Predictors of upper trapezius pain with myofascial trigger points in food service workers: the STROBE study. Medicine (Baltimore) 2017; 96: e7252.

18. Shah JP, Danoff JV, Desai MJ, Parikh S, Nakamura LY, Phillips TM, et al. Biochemicals associated with pain and inflammation are elevated in sites near to and remote from active myofascial trigger points. Arch Phys Med Rehabil 2008; 89: 16-23.

19. Xie P, Qin B, Song G, Zhang Y, Cao S, Yu J. Microstructural abnormalities were found in brain gray matter from patients with chronic myofascial pain. Front Neuroanat 2016; 10: 122.

20. Grosman-Rimon L, Parkinson W, Upadhye S, Clarke H, Katz J, Flannery J, et al. Circulating biomarkers in acute myofascial pain: a case-control study. Medicine (Baltimore) 2016; 95: e4650.

21. Niddam DM, Lee SH, Su YT, Chan RC. Brain structural changes in patients with chronic myofascial pain. Eur J Pain 2017; 21: 148-158.

22. Fernández-de-Las-Peñas C, Nijs J. Trigger point dry needling for the treatment of myofascial pain syndrome: current perspectives within a pain neuroscience paradigm. J Pain Res 2019; 12: 1899-1911.

23. Cerezo-Téllez E, Torres-Lacomba M, Mayoral-del Moral O, Sánchez-Sánchez B, Dommerholt J, GutiérrezOrtega C. Prevalence of myofascial pain syndrome in chronic non-specific neck pain: a population-based crosssectional descriptive study. Pain Med 2016; 17: 2369-2377.

24. Koca İ, Tutoglu A, Boyacı A, Pehlivan Y, Yıldız H, Turkbeyler I, et al. An evaluation of oxidative stress and antioxidant capacity in patients with myofascial pain syndrome. Mod Rheumatol 2014; 24: 992-996.

25. Holanda L, Fernandes A, Cabral AC, Santos Junior F. Pathophysiology of myofascial trigger points: a review of literature. Int J Basic Appl Sci 2014; 4: 73-77.

26. Quintner JL, Bove GM, Cohen ML. A critical evaluation of the trigger point phenomenon. Rheumatology 2015; 54: 392-399.

27. Jadoon S, Malik A. A review article on the formation, mechanism and biochemistry of MDA ans MDA as a biomarker of oxidative stress. Int J Adv Res 2017; 5: 811-818.

28. Singh Z, Karthigesu IP, Singh P, Kaur R. Use of malondialdehyde as a biomarker for assessing oxidative stress in different disease pathologies: a review. Iran J Public Health 2014; 43: 11

29. Bakirezer SD, Yaltirik CK, Kaya AH, Yilmaz SG, Ozdogan S, Billur D, et al. The evaluation of glutathione reductase and malondialdehyde levels in patients with lumbar disc degeneration disease. In Vivo 2019; 33: 811-814.

30. Ayala A, Muñoz MF, Argüelles S. Lipid peroxidation: production, metabolism, and signaling mechanisms of malondialdehyde and 4-hydroxy-2-nonenal. Oxid Med Cell Longev 2014; 2014: 360438. DOI: HTTPs://DOI. ORG/10.1155/2014/360438.

31. Medeiros LF, Caumo W, Dussán-Sarria J, Deitos A, Brietzke A, Laste G, et al. Effect of deep intramuscular stimulation and transcranial magnetic stimulation on neurophysiological biomarkers in chronic myofascial pain syndrome: neuromodulation techniques and biomarkers. Pain Med 2016; 17: 122-135.

32. Gomez I, Foudi N, Longrois D, Norel X. The role of prostaglandin E2 in human vascular inflammation. Prostaglandins Leukot Essent Fatty Acids 2013; 89: 55-63.

33. Su YS. Molecular mechanism of inflammatory pain. World J Anesthesiol 2014; 3: 71.

34. Sugita R, Kuwabara H, Kubota K, Sugimoto K, Kiho T, Tengeiji A, et al. Simultaneous inhibition of PGE 2 and PGI 2 signals is necessary to suppress hyperalgesia in rat inflammatory pain models. Mediators Inflamm 2016; 2016: 9847840.

35. Yam M, Loh Y, Tan C, Khadijah Adam S, Abdul Manan N, Basir R. General pathways of pain sensation and the major neurotransmitters involved in pain regulation. Int J Mol Sci 2018; 19: 2164.

36. Li QB, Chang L, Ye F, Luo QH, Tao YX, Shu HH. Role of spinal cyclooxygenase-2 and prostaglandin E2 in fentanyl-induced hyperalgesia in rats. Br J Anaesth 2018; 120: 827-835.

37. Morimoto K, Shirata N, Taketomi Y, Tsuchiya S, Segi-Nishida E, Inazumi T, et al. Prostaglandin E2-EP3 signaling induces inflammatory swelling by mast cell activation. J Immunol 2014; 192: 1130-1137.

38. Odendaal C. The management of myofascial pain syndrome. South Afr J Anaesth Analg 2003; 9: 19-24.

39. Domenichiello AF, Wilhite BC, Keyes GS, Ramsden CE. A dose response study of the effect of prostaglandin E2 on thermal nociceptive sensitivity. Prostaglandins Leukot Essent. Fatty Acids 2017; 126: 20-24.

40. Li T, Chen X, Zhang C, Zhang Y, Yao W. An update on reactive astrocytes in chronic pain. J Neuroinflammation 2019; 16: 140

41. Ji RR, Berta T, Nedergaard M. Glia and pain: is chronic pain a gliopathy? Pain 2013; 154: S10-S28.

42. Ren K. Emerging role of astroglia in pain hypersensitivity. Jpn Dent Sci Rev 2010; 46: 86-92.

43. Nam Y, Kim JH, Kim JH, Jha MK, Jung JY, Lee MG, et al. Reversible induction of pain hypersensitivity following optogenetic stimulation of spinal astrocytes. Cell Rep 2016; 17: 3049-3061.

44. Liddelow SA, Barres BA. Reactive astrocytes: production, function, and therapeutic potential. Immunity 2017; 46: 957-967. 